\title{
Tipos de tratamientos prescritos en un grupo de pacientes diabéticos en una farmacia comunitaria
}

\author{
Eva Sáez-Fernández', Ana Risoto-Baena², Amalia I. Pozas-Guerrero ${ }^{3}$, Laura Cabrera-Castilla ${ }^{3}$, \\ Sara Cejudo-Nieto ${ }^{2}$, Felisa Baena-Mira ${ }^{2}$ \\ 1. Doctora en Ciencias Farmacéuticas. 2. Licenciada en Farmacia. 3. Auxiliar.
}

PALABRAS CLAVE

Diabetes, metformina, Plan Integral de Diabetes

\section{ABREVIATURAS}

DM: diabetes mellitus

PID: Plan Integral de Diabetes

\section{KEYWORDS}

Diabetes, metformin, Plan Integral de Diabetes

\section{RESUMEN}

Objetivo: conocer el porcentaje de pacientes que tienen prescrito tratamiento para la diabetes mellitus, tipo de tratamiento y comparativa con el Plan Integral de Diabetes de Andalucía.

Métodos: se incluyó en el estudio a todos aquellos pacientes que accedieron a la Farmacia Felisa Baena de Linares (Jaén) y quisieron facilitar su tratamiento completo $(\mathrm{n}=500)$. A través de un estudio observacional se comprobó el tipo de tratamiento y su coincidencia con el Plan Integral de Diabetes.

Resultados: se comprobó que el 19,2\% de los pacientes objeto de estudio presentaban diabetes, de estos, el $75 \%$ solo estaba en tratamiento por vía oral y el $9 \%$ solo con insulina. De los resultados obtenidos se comprobó que un $80 \%$ de los pacientes tenía prescrita metformina ya fuese en monoterapia, en combinación con otros antidiabéticos orales o con insulina. Del total de los pacientes con diabetes solo el $1 \%$ tenía prescrito glucagón y un $16 \%$ tiras reactivas, muy por debajo de lo establecido en el Plan Integral de Diabetes, que debería haberse encontrado en este caso en un $47 \%$ de los pacientes.

Conclusiones: en nuestro entorno existe un alto número de pacientes con diabetes, predominando la terapia con metformina. Hay un bajo porcentaje de pacientes que tiene prescrito glucagon y tiras reactivas, comparado con el Plan Integral de Diabetes, lo que supone un riesgo para la salud del paciente. De aquí la importancia de desarrollar programas de salud desde la farmacia para que los pacientes conozcan los controles de la diabetes.

Types of treatments prescribed in a group of diabetic patients in a community pharmacy

\section{ABSTRACT}

Objective: To determine the percentage of patients who are prescribed treatment for diabetes mellitus, type of treatment and comparison with the Plan Integral Diabetes (Junta Andalucía).

Methods: The study included all patients who accessed the Felisa Baena Pharmacy Linares (Jaen) and wanted to facilitate complete treatment $(n=500)$. Through an observational study the type of treatment of patients and the relationship with the Plan Integral Diabetes.

Results: It was found that $19.2 \%$ of patients in the study had diabetes, of these, $75 \%$ were only treatable orally and $9 \%$ only with insulin. From the results obtained it was found that $80 \%$ of patients were prescribed metformin either in monotherapy, in combination with other oral agents or insulin. Of all patients with diabetes only $1 \%$ had prescribed glucagon and 16\% test strips, well below the provisions of the Plan Integral Diabetes, which should have been in this case $47 \%$ of patients.

Conclusions: In our environment there is a high number of patients with diabetes, metformin therapy predominating. There is a low percentage of patients who have been prescribed glucagon and test strips compared with the Plan Integral Diabetes, which poses a risk to patient health. Hence the importance of developing health programs from the pharmacy so that patients are aware how to control diabetes.

Financiación: ninguna.

Conflicto de intereses: ninguno.

Cite este artículo como: Sáez-Fernández E Risoto-Baena A, Pozas-Guerrero Al, Cabrera-Castilla L Cejudo-Nieto S, Baena-Mira F. Tipos de tratamientos prescritos en un grupo de pacientes diabéticos en una farmacia comunitaria. Farmacéuticos Comunitarios. 2020 Mar 06;12(1):17-21. doi:10.5672/FC.2173-9218.(2020/Vol12).001.03

Correspondencia: Eva Sáez-Fernández (evasaez86@hotmail.com).

ISSN 1885-8619 @SEFAC (Sociedad Española de Farmacia Familiar y Comunitaria). Todos los derechos reservados. 


\section{Introducción}

La diabetes mellitus (DM) es un grupo de enfermedades crónicas que afectan a un gran número de personas y se caracteriza por la presencia de niveles de azúcar en sangre elevados. Nos centramos en la diabetes tipo 2. En nuestro país el $13,8 \%$ de la población adulta la padece, de entre los cuales un $43 \%$ desconocía que padecía la enfermedad (1). Se estima que la prevalencia de diabetes a nivel mundial se incrementará un $72 \%$ entre los años 2010 y 2030 (1). Además, se calcula que hay un alto número de pacientes en estado pre-diabético que deberían ser controlados para evitar la aparición de complicaciones (2).

Como es bien conocido, la DM tipo 2 se produce por una compleja alteración metabólica que se caracteriza por una alteración en la secreción y/o en la acción de la insulina, que da lugar a un incremento de la glucosa en sangre (3-4).

La hiperglucemia crónica afecta a largo plazo a diferentes órganos, particularmente a los ojos, al riñón, a los vasos sanguíneos, al sistema nervioso y al corazón. Puede producir retinopatías que cursan con ceguera, nefropatías que pueden desencadenar en un fallo renal, neuropatía periférica con posibles complicaciones que conllevan a la amputación de la zona afectada y enfermedades cardiovasculares como cardiopatías isquémicas y accidentes cerebrovasculares, entre otras (5).

Para el tratamiento farmacológico de la DM tipo 2 existen varios grupos terapéuticos de antidiabéticos orales e insulina, cuyas características principales se recogen en la tabla 1 . Suele iniciarse el tratamiento con monoterapia y si no hay un control adecuado de la glucemia se considera una de las múltiples opciones de combinación de tratamiento (6-7).

En pacientes con tratamiento farmacológico la complicación más frecuente es la hipoglucemia. Se caracteriza por una concentración de glucosa en sangre venosa inferior a $60 \mathrm{mg} / \mathrm{dl}$ o capilar inferior a $50 \mathrm{mg} /$ $\mathrm{dl}$, aunque hay veces que no resulta útil esta definición, pues hay pacientes que pueden sufrir deterioros neurológicos con concentraciones ligeramente superiores a las indicadas.
Podemos diferenciar entre hipoglucemia leve (se perciben síntomas ligeros de ansiedad, taquicardia...), moderada (confusión, conducta inadecuada...) y grave (coma, crisis convulsivas...). Para revertirlo es necesario el consumo de hidratos de carbono de absorción rápida, glucosa pura o administrar glucagón inyectable que produzcan un aumento rápido de la glucosa en sangre (8). De aquí la importancia de que los pacientes que a priori puedan estar en riesgo de sufrir hipoglucemia tengan prescrito este medicamento, que lo conserven en casa en las condiciones óptimas de almacenaje y, lo más importante, que sepan utilizarlo.

Desde la Junta de Andalucía se ha considerado la diabetes como un problema de salud prioritario, y es por ello que desde 2003 ya se contemplaba el realizar un Plan Integral de Diabetes (PID) (9) con la finalidad de coordinar mejor los recursos disponibles y las actuaciones sanitarias en torno a la diabetes. Se desarrolla el primer PID a partir de 2003, y desde entonces se propone en el mismo la implantación completa del proceso

Tabla 1 Caracteristicas de los grupos terapéuticos de tratamientos antidiabéticos

\begin{tabular}{|c|c|c|c|c|c|c|c|}
\hline & Biguanidas & Tiazolidindionas & $\begin{array}{l}\text { Secretagogos, } \\
\text { glinidas y } \\
\text { sulfonilureas }\end{array}$ & $\begin{array}{l}\text { Inhibidores } \\
\text { de DPP-4 }\end{array}$ & $\begin{array}{l}\text { Inhibidores } \\
\text { selectivos } \\
\text { de SGLT-2 }\end{array}$ & $\begin{array}{l}\text { Análogos } \\
\text { de GLP-1 }\end{array}$ & $\begin{array}{l}\text { Insulina } \\
\text { basal }\end{array}$ \\
\hline $\begin{array}{l}\text { Reducción de } \\
\mathrm{HbA1c}\end{array}$ & Alta $(1,3-2,0 \%)$ & Alta $(0,8 \%)$ & Alta & Media & Alta & Alta & Alta (1-2 \%) \\
\hline $\begin{array}{l}\text { Riesgo de } \\
\text { Hipoglucemia }\end{array}$ & Bajo & Bajo & $\begin{array}{c}\text { Gliclazida: ligero } \\
\text { a moderado; } \\
\text { Glimepirida: } \\
\text { Moderado; } \\
\text { Glibenclamida: } \\
\text { Alto }\end{array}$ & Bajo & Bajo & Bajo & Alto \\
\hline Peso & Efecto neutro & Efecto neutro & $\begin{array}{c}\text { Aumento medio } \\
2.31 \mathrm{~kg}\end{array}$ & No altera el peso & $\begin{array}{c}\text { Ligera } \\
\text { disminución }\end{array}$ & $\begin{array}{l}\text { Disminución } \\
\text { considerable }\end{array}$ & $\begin{array}{c}\text { Subida } \\
\text { considerable } \\
\text { hasta } 6 \mathrm{~kg}\end{array}$ \\
\hline $\begin{array}{l}\text { Uso en } \\
\text { insuficiencia } \\
\text { renal }\end{array}$ & $\begin{array}{l}\text { No usar por } \\
\text { debajo } 45 \mathrm{ml} / \\
\text { min Filtración } \\
\text { Glomerular } \\
\text { estimada (FGE) }\end{array}$ & $\begin{array}{l}\text { No ajuste de } \\
\text { dosis }\end{array}$ & $\begin{array}{l}\text { Suspender si FGE } \\
<30 \mathrm{ml} / \mathrm{min}\end{array}$ & Ajuste de dosis & $\begin{array}{l}\text { Suspender si } \\
\text { FGE }<45 \mathrm{ml} / \\
\quad \min \end{array}$ & $\begin{array}{l}\text { Suspender si } \\
\text { FGE }<30 \mathrm{ml} / \\
\text { min }\end{array}$ & $\begin{array}{l}\text { No tiene } \\
\text { limitación }\end{array}$ \\
\hline $\begin{array}{l}\text { Efecto } \\
\text { secundarios }\end{array}$ & $\begin{array}{l}\text { Gastrointestinales } \\
\text { Ácido láctico } \\
\text { Déficit Vit B12 }\end{array}$ & $\begin{array}{l}\text { Edema } \\
\text { IC } \\
\text { Otros }\end{array}$ & $\begin{array}{l}\text { Agotamiento } \\
\text { Pancreático }\end{array}$ & $\begin{array}{c}\text { GI } \\
\text { Pancreatitis } \\
\text { IC }\end{array}$ & $\begin{array}{l}\text { IGU } \\
\text { Otros }\end{array}$ & $\begin{array}{c}\text { GI } \\
\text { Pancreatitis }\end{array}$ & --- \\
\hline $\begin{array}{l}\text { Riesgo } \\
\text { Cardiovascular }\end{array}$ & Bajo & Bajo & Alto & $\begin{array}{c}\text { Seguridad } \\
\text { cardiovascular }\end{array}$ & Muy bajo & Muy bajo & $\begin{array}{c}\text { Seguridad } \\
\text { cardiovascular }\end{array}$ \\
\hline Complejidad & Bajo & Baja & Baja & Baja & Media & Alta & Alta \\
\hline Coste & Muy Bajo & Bajo & Muy bajo & Medio & Medio & Muy alto & Alta \\
\hline
\end{tabular}


asistencial, la educación para la salud, una guía de elección de tratamiento según el paciente, se especifica qué pacientes deben realizar un autoanálisis de la glucemia capilar y qué pacientes deben tener siempre glucagón, entre otras recomendaciones.

El primer PID tuvo por objetivo reducir la incidencia y el impacto de la diabetes en Andalucía, mejorando la atención sanitaria, la oferta de servicios a la población, aumentando el grado de conocimiento e información sobre diabetes en Andalucía... Cuando se evaluó, se comprobó que es una estrategia válida y efectiva en la mejora de la atención a la persona con diabetes.

Con la información obtenida se elaboró el II PID, en donde se establecen una serie de áreas de mejora y se planifican estrategias orientadas de forma específica a la prevención de diabetes y sus complicaciones, atención sanitaria, educación terapéutica, colaboración con asociaciones de ayuda mutua... y se establecen unas revisiones mínimas que se deben realizar al paciente:

- Control del peso, tensión arterial... $\rightarrow$ cada 3 meses.

- Hemoglobina glicosilada $\rightarrow$ cada 6 meses.

- Analítica completa $\rightarrow 1$ al año.

- Electrocardiograma $\rightarrow 1$ al año.

- Revisiones de pies $\rightarrow$ mínimo 1 al año.

- Retinografía $\rightarrow$ mínimo 1 cada 3 años.

Además, como proceso asistencial recoge como medicamento de primera elección la metformina (siempre que el paciente no presente cualquier tipo de intolerancia a la misma) y de segunda elección a las sulfonilureas, señalando que siempre que un paciente tenga prescritas insulinas o sulfonilureas deberá tener prescrito glucagón, que el paciente debe guardar en casa y se tendrá que formar al cuidador del mismo para que pueda hacer uso de la misma si hubiese una hipoglucemia.

El presente trabajo tiene como objetivo realizar un estudio observacional que permita conocer el porcentaje de pacientes que tienen prescrito tratamiento para la DM tipo 2 dentro de un grupo de pacientes seleccionados al azar. Conocer el tipo de tratamiento prescrito para cada uno de los pacientes y comparar con lo establecido en el Plan Integral de Diabetes de Andalucía.

\section{Métodos}

El presente trabajo se ha desarrollado en la Farmacia Felisa Baena Mira de Linares (Jaén). Es una farmacia de paso, situada en una zona céntrica de la ciudad y que tiene un gran número de clientes fidelizados.

Se decidió que la toma de datos de pacientes se realizaría entre los meses de enero y marzo de 2019, intentando conseguir al menos 450 pacientes, ya que se consideró que a partir de este valor se podría obtener un valor significativo de pacientes con diabetes dentro de la población general del estudio. Esto supuso al menos 7 pacientes al día durante los 3 meses, seleccionando los 4 pacientes que accediesen primero en el turno de mañana y los 3 pacientes que accediesen en primer lugar en el turno de tarde (siempre que quisiesen participar).

En el estudio se incluyeron todos aquellos pacientes que accedieron a participar en él, facilitando toda la medicación que tenían prescrita y que tomaban (sin recoger en ningún momento datos personales o datos que pudiesen relacionar los tratamientos registrados con el paciente en cuestión). Se excluyeron aquellos pacientes cuya medicación es retirada por otra persona, así como pacientes con algún problema psíquico o físico que les impida responder a la información solicitada y mujeres embarazadas con diabetes gestacional.

Se realizó el estudio de cada uno de los medicamentos de los pacientes a través de la realización de una base de datos en la que se recogían uno por uno los mismos (tanto pacientes que estén diagnosticados como diabéticos como no diagnosticados). Del total de pacientes que se consigue recoger datos, se seleccionaron aquellos pacientes que tenían prescrito algún medicamento para la DM y presentaban DM tipo 2.

Se realizó un estudio observacional, en el que se estudió el tipo de medicamentos prescritos, número de medicamentos y la combinación de principios activos que se recogían en los tratamientos de pacientes para dicha patología.

Tras el estudio del PID, se ejecutó una comparativa para ver si se cumplía con las indicaciones sobre medicamentos recogidas en el mismo.

\section{Resultados}

El número de pacientes que accedió a participar en el estudio fue de 500. A todos ellos se les recogió el tratamiento completo. De estos, el 19,2 \% estaba en tratamiento con medicamentos para la DM tipo 2, concretamente 96 pacientes.

En la figura 1 se recoge la distribución del tratamiento de los pacientes estudiados, diferenciando entre los pacientes que solo tenían prescripción por vía oral (diferenciando entre los que toman solamente un tipo de principio activo o varios principios activos), aquellos pacientes que tomaban antidiabéticos orales, pero también inyectables y los pacientes que tenían tratamiento con insulina (diferenciando solo insulina e insulina con tratamiento oral). Se pudo comprobar que el 91,0\% de los pacientes tenía prescrito tratamiento por vía oral, aunque en ocasiones podían ir asociado con insulina o antidiabéticos inyectables.

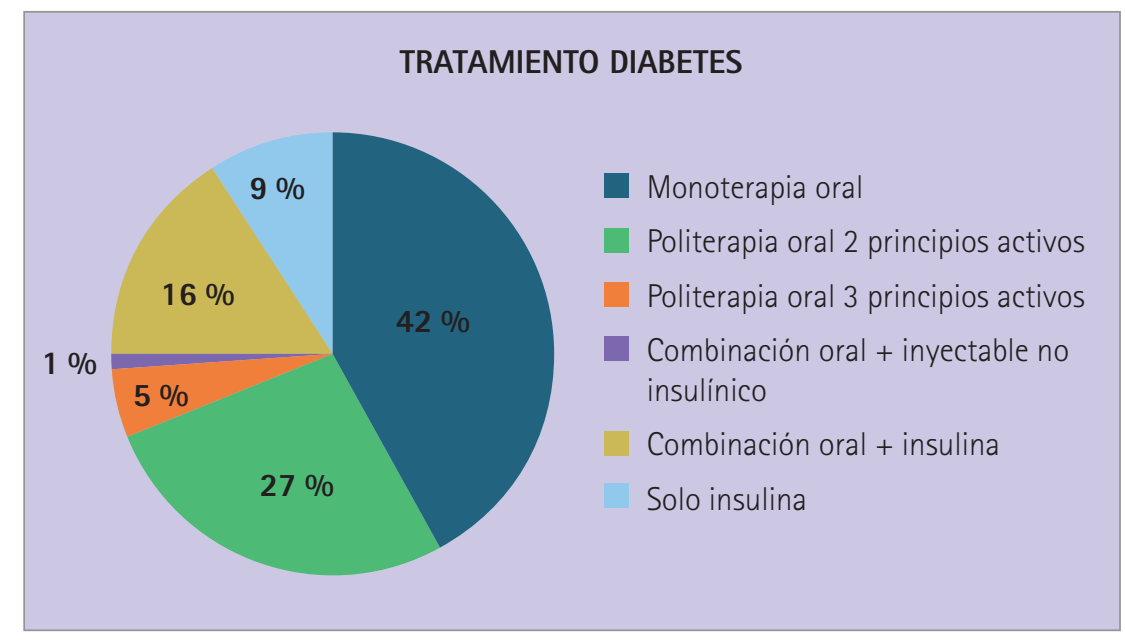

Figura 1 Distribución del tipo de tratamiento prescrito a los pacientes del estudio 


\section{DISTRIBUCIÓN DE PRESCRIPCIÓN DE PRINCIPIO ACTIVO VÍA ORAL}

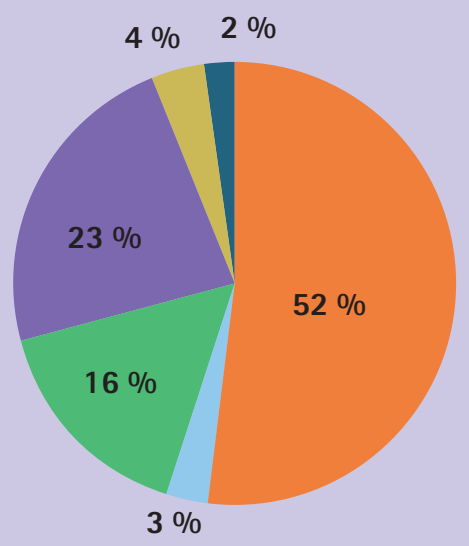

Figura 2 Distribución de grupos terapéuticos en terapia oral

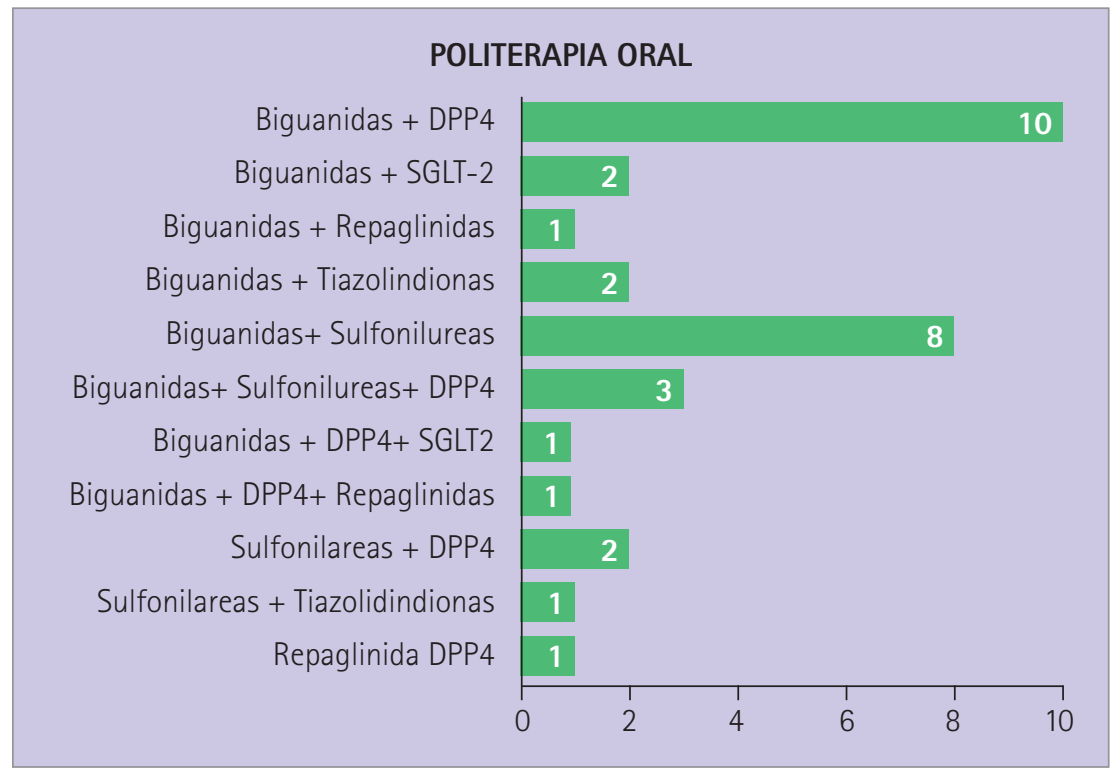

Figura 3 Distribución de politerapia oral según grupos terapéuticos combinados

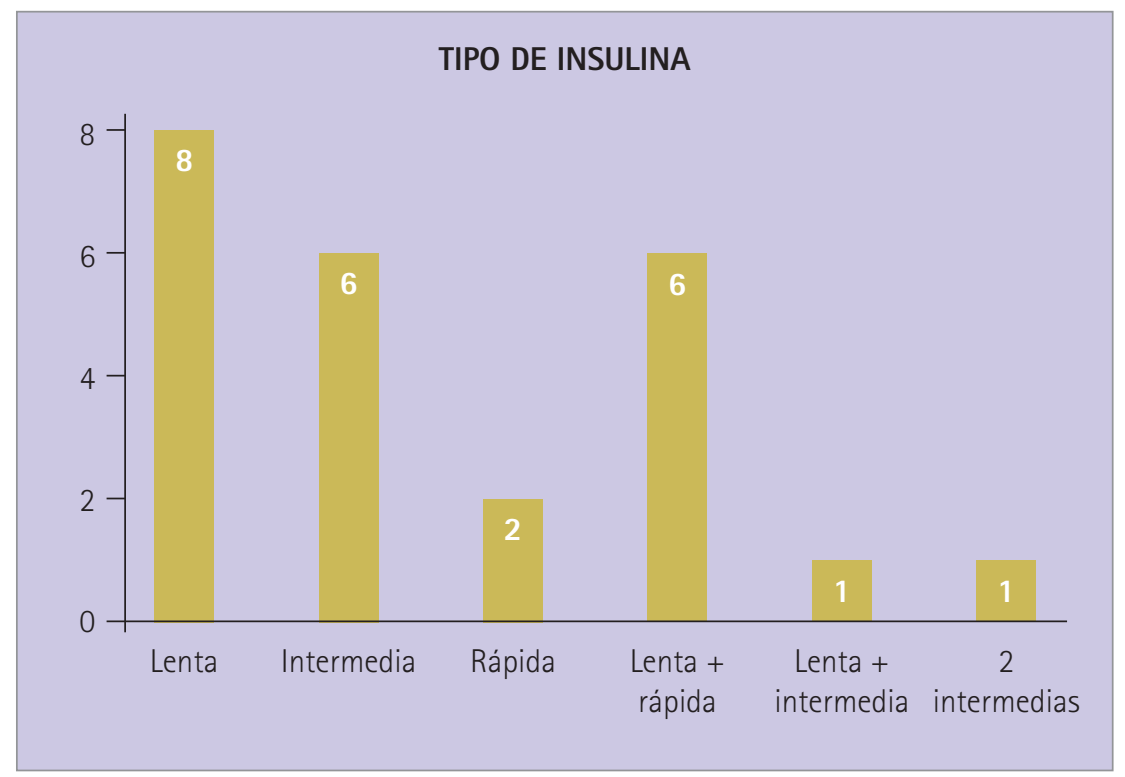

Figura 4 Distribución del tipo de insulinas prescritas
En la figura 2, se recoge una distribución de los grupos terapéuticos orales que se han prescrito.

Se encontró que había un 75,0\% de los pacientes que no estaba en tratamiento con insulina. De estos, un $1,0 \%$ tenía prescrito antidiabéticos orales e inyectables del grupo GLP-1.

La distribución según el grupo terapéutico, diferenciando entre tratamientos combinados y monoterapia, fue la siguiente: a) tratamiento con monoterapia: solo tenían prescrito un principio activo ( $41 \%$ de los pacientes con diabetes), de los cuales el 79,5\% utilizaba metformina (grupo biguanidas), el 12,8 \% tenía prescritas sulfonilureas y el 7,7\% medicamentos del grupo de los DPP-4; b) tratamiento combinado: un 33,0 \% de los pacientes con diabetes tomaba tratamiento combinado por vía oral; de estos señalar que el grupo más utilizado en combinación es el de las biguanidas, presente en un $87,5 \%$ de los casos. En la figura 3 se indica la combinación de principios activos que se recogen en las prescripciones de los pacientes objeto de estudio.

El 25,0\% restante del total de pacientes con DM tenían prescrita insulina. La clasificación de los tratamientos se divide de la siguiente forma: a) tratamiento con antidiabéticos orales e insulina: fue el grupo más numeroso dentro de los pacientes insulinodependientes, correspondiendo con un $62,5 \%$; $b$ ) tratamiento con dos insulinas: un 25,0\% de los pacientes; c) solo tenían prescrita una insulina: un 8,3 \% de los pacientes; y, d) tratamiento con tres insulinas diferentes: 4,2 \% de los pacientes.

En la figura 4 se recoge la distribución según el tipo de insulinas que tenían los pacientes prescritos.

\section{Discusión}

En nuestro estudio el 19,2\% de los pacientes entrevistados utilizaba medicamentos para la DM tipo 2. El resultado es un poco superior al encontrado en Andalucía, donde la prevalencia de diabetes es del 15,3\%, y al de España, donde el valor es de $13,8 \%$.

De los resultados obtenidos se comprobó que el $80 \%$ de los pacientes con DM tipo 2 tienen prescrita metformina, coincidiendo de esta manera el tratamiento de elección 
con el seleccionado en el PID (8-9), donde se recomienda la metformina como primera terapia farmacológica en los pacientes con diabetes tipo $2 \mathrm{y}$ en el caso de que este tratamiento no funcione se irán realizando diferentes combinaciones, tal como se comprobó en el estudio realizado.

En el caso de pacientes con DM tipo 2 insulinizados, la insulina más utilizada es la lenta sola o en combinación con una insulina rápida, para evitar los picos de glucemia como consecuencia de una rápida actuación de la misma (no se conoce la pauta establecida).

El PID recoge también que aquellos pacientes que presenten DM tipo 2 insulinizados y pacientes con antidiabéticos orales que puedan producir hipoglucemias por su gran capacidad para disminuir la glucosa en sangre (como las sulfonilureas) deben de realizarse autoanálisis para conocer el valor de la glucemia capilar y controlar la posible aparición de hipoglucemias. Se comprobó que, según los resultados obtenidos, no se cumple con lo establecido, ya que de los pacientes con DM tipo 2 insulinizados solo el $54 \%$ tenía prescritas tiras reactivas, y de los pacientes con tratamiento vía oral solo el $3 \%$; un porcentaje muy bajo teniendo en cuenta que el $29 \%$ de los pacientes estaba en tratamiento con sulfonilureas.

Solo el $1 \%$ de los pacientes se encontraba en tratamiento con glucagón, lo que suponía otra discordancia con respecto al PID, donde se establece que se debe prescribir a personas en tratamiento con insulina y/o con riesgo de hipoglucemia grave $\mathrm{y}$, además, se debe enseñar cómo utilizarlo a los familiares o cuidadores.

Por lo tanto, del total de los pacientes con diabetes solo el $1 \%$ tenía prescrito glucagón y un $16 \%$ tiras reactivas, muy por debajo de lo establecido en el Plan Integral de Diabetes, donde establecen que todos los pacientes con insulina o tratamientos con sulfonilurea y meglitinidas deben tenerlo prescrito por el riesgo a sufrir hipoglucemia. Este porcentaje debería estar en un $47 \%$ de los pacientes.

Sería muy interesante realizar planes de formación para los pacientes con diabetes en las farmacias comunitarias, para que el paciente conozca la importancia del autoanálisis capilar, el uso de glucagón por parte de los familiares o cuidadores de los pacientes en riesgo de sufrir hipoglucemia y que todos conozcan las revisiones que deben de realizarles los facultativos según se establece en el Plan Integral de Diabetes.

\section{Conclusiones}

Entre los pacientes de la Farmacia Felisa Baena de Linares hay un alto número de pacientes con tratamiento para la diabetes, siendo el fármaco más utilizado la metformina por su bajo coste y complejidad, cumpliendo con el de elección que se ha establecido en el Plan Integral de Diabetes de la Junta de Andalucía.

Hay una muy baja prescripción de glucagón y de tiras para la medida de glucosa por parte de los profesionales, incumpliendo lo establecido por el Plan Integral de Diabetes y poniendo en riesgo la aparición de hipoglucemia en los pacientes.

\section{Agradecimientos}

Agradecimiento a D. José L. Arias por sus consejos y ayuda incondicional. También queremos agradecer a todos nuestros pacientes por participar y facilitarnos sus tratamientos.

\section{Referencias bibliográficas}

1. Shaw JE, Sicree RA, Zimmet PZ. Global estimates of the prevalence of diabetes for 2010 and 2030. Diabetes Res Clin Prac. 2010; 87: 4-14. doi:10.1016/j.diabres.2009.10.007

2. Soriguer F, Goday A, Bosch-Comas A et al. Prevalence of diabetes mellitus and impaired glucose regulation in Spain: the Di@bet.es Study. Diabetologia. 2012; 55: 88-93. doi:10.1007/ s00125-011-2336-9

3. Qi Q, Hu FB. Genetics of type 2 diabetes in European populations. J Diabetes. 2012; 4: 203-212. doi:10.1111/j.17530407.2012.00224.X

4. Bi Y, Wang T, Xu M, et al. Advanced researc on risk factors of type 2 diabetes. Diabetes Metab Res Rev. 2012; 28 (2): 32-39. doi:10.1002/dmrr.2352

5. Vitimilla enderica PF, Giler Mendoza YO, Motoche Apolo KE, Ortega Flores JJ. Diabetes Mellitus Tipo 2: Incidencias, Complicaciones y Tratamientos actuales. Revista Científica de la Investigación y el Conocimiento. 2019; 3(1): 26-37. doi:10.26820/recimundo/3.(1). enero.2019.26-37

6. Spanish Diabetes Society (SED) recommendations for the pharmacologic treatment of hyperglycemia in type 2 diabetes: 2018 update. Gómez-Peralta F, Escalada San Martín FJ, Menendez Torre E, et al. Endocrinol Diabetes Nutr. 2018; 65 (10): 611-624. doi:10.1016/j. endien.2018.11.010

7. Martinez Broca M Asunción et al. Diabetes Mellitus: proceso asistencial integrado. Consejeria de Educación. 2018 [acceso mayo 2019] Disponible en: https://www.juntadeandalucia.es/export/drupaljda/diabetes_ mellitus_2018_18_06_2018.pdf

8. Mediavilla Bravo JJ. Complicaciones de la diabetes mellitus. Diagnóstico y tratamiento. SEMERGEN. 2001; 27 (3): 132145. doi:10.1016/S1138-3593(01)73931-7

9. Aguilar Diosdado Manuel et al. Proceso asistencial integrado Diabetes Mellitus. Junta de Andalucía. Consejería de Salud. 2011 [acceso mayo 2019]. Disponible en: https://www.juntadeandalucia.es/export/ drupaljda/salud_5af1956f2c254_diabetes_mellitus_2011.pdf 